\title{
Serum SRANKL and SRANKL/OPG ratio: Novel biomarkers in non-small cell lung cancer
}

\author{
CHENGJUN LU ${ }^{1 *}, \mathrm{CHAO} \mathrm{SUN}^{2^{*}}$ and HAI JIN ${ }^{1}$ \\ ${ }^{1}$ Department of Cardiothoracic Surgery, Changhai Hospital of Shanghai Affiliated to The Second Military Medical University, \\ Shanghai 200433; ${ }^{2}$ Hematology Department, Wuxi People's Hospital, Affiliated to Nanjing Medical University, \\ Nanchang, Wuxi 214023, P.R. China
}

Received August 19, 2014; Accepted May 5, 2015

DOI: $10.3892 / \mathrm{ol} .2016 .4166$

\begin{abstract}
Osteoprotegerin(OPG) and soluble receptor activator of nuclear factor- $\kappa \mathrm{B}$ ligand (sRANKL) are bone-regulating molecules. The two molecules have each been indicated to be involved in carcinogenesis. However, the diagnostic significance in non-small cell lung cancer (NSCLC) remains to be investigated. Thus, the objective of the present study was to investigate the serum levels of OPG and sRANKL in NSCLC patients, and to analyze their clinical significance. Serum OPG and sRANKL levels were determined in 50 patients with NSCLC, matched with 25 patients with benign lung nodule and 25 healthy controls by enzyme-linked immunosorbent assay. The level of serum sRANKL and the sRANKL/OPG ratio were significant elevated in the patients with NSCLC compared with the benign lung nodule patients and the healthy controls. Receiver operating characteristic curves were used to evaluate the performance of sRANKL and sRANKL/OPG. When the cut-off values for the sRANKL level and the sRANKL/OPG ratio were set at $4.20 \mathrm{pmol} / 1$ and 0.60 , respectively, the sensitivity, specificity and accuracy of sRANKL were 74.0, 84.0 and $77.3 \%$, respectively. Moreover, the sensitivity, specificity and accuracy of the sRANKL/OPG ratio were 84.0, 88.0 and $85.3 \%$, respectively. On the other hand, when the cut-off values of the serum sRANKL level and the sRANKL/OPG ratio were set at $5.24 \mathrm{pmol} / 1$ and 0.63 , respectively, the sensitivity, specificity and accuracy of sRANKL were 60.0, 84.0 and $68.0 \%$, respectively. The sensitivity, specificity and accuracy
\end{abstract}

Correspondence to: Dr Hai Jin, Department of Cardiothoracic Surgery, Changhai Hospital of Shanghai Affiliated to The Second Military Medical University, 168 Changhai Road, Yangpu, Shanghai 200433, P.R. China

E-mail: haijin4043@163.com

${ }^{*}$ Contributed equally

Key words: non-small cell lung cancer, osteoprotegerin, receptor activator for nuclear factor- $\kappa \mathrm{B}$ ligand, serum of the ratio were $78.0,64.0$ and $73.3 \%$, respectively. The OPG/RANKL system may be involved in the pathogenesis of NSCLC. More importantly, the serum sRANKL level and the sRANKL/OPG ratio may have the potential to be novel biomarkers for the diagnosis of NSCLC.

\section{Introduction}

With regard to the global incidence of cancer, lung cancer is currently ranked first and is the leading cause of cancer-related mortality, with an overall 5-year survival rate of only $17 \%(1,2)$. This malignant tumor occurs with an annually increasing trend. Non-small cell lung cancer (NSCLC) accounts for $\sim 80 \%$ of all lung cancer types (3). NSCLC can be classified as two major histological subtypes: Adenocarcinoma (AC) and squamous cell carcinoma (SCC) (4). Approximately $65-75 \%$ of patients with NSCLC possess unresectable advanced or metastatic disease at diagnosis (5). However, there remains a lack of an effective biomarker to diagnosis the malignant disease at an early stage. Furthermore, NSCLC presents with insidious onset, but with a high level of malignancy and fast progression (2). Thus, the most viable method by which to improve the 5-year survival rate in NSCLC patients is early detection, and subsequent effective treatment.

A novel receptor-ligand system belonging to the tumor necrosis factor (NF) superfamily, termed receptor activator of nuclear factor $(\mathrm{NF})-\kappa \mathrm{B}$ ligand (RANKL) and osteoprotegerin (OPG), has recently become a focus of attention (6). RANKL is a membrane-bound protein that is expressed mainly on the surface of osteoblasts and bone marrow stromal cells, and binds to RANK on the surface of osteoclast precursors, thus stimulating their differentiation into mature osteoclasts (7). The RANKL/OPG system was the first pathway to be found to be involved in bone regulation (8). More significantly, recent studies have indicated that this system is also important in the pathogenesis of lung cancer, particularly the regulation of bone tumor metastasis $(9,10)$. The present study aimed to determine the levels of OPG and soluble RANKL (sRANKL) in NSCLC patients, to investigate the diagnostic significance of the sRANKL level and the RANKL/OPG ratio, and to further analyze the role of this system in the progression of NSCLC. 


\section{Materials and methods}

Subjects. A total of 50 NSCLC patients who underwent a radical resection for lung cancer between October 2011 and February 2013 were enrolled in the present study. All subjects were classified pathologically with SCC (20 cases) or AC (30 cases) (11). The patients with diseases such as liver and kidney disease, heart failure, diabetes, lupus and rheumatoid arthritis, as well as those with fractures, were excluded. Furthermore, 25 patients with benign space-occupying pulmonary lesions were also enrolled. The detailed medical histories, including smoking history and medical history relevant to exclusion criteria, were retrieved for all enrolled patients. The pathological results in the NSCLC patients and the patients with benign space-occupying pulmonary lesions were confirmed by the Department of Pathology in the Changhai Hospital of Shanghai Affiliated to the Second Military Medical University (Shanghai, China). Moreover, 25 healthy subjects who were examined and found to be without any disease were also enrolled as controls. This study was approved by the Ethics Committee of the First Affiliated Hospital of Nanjing Medical University (Nanchang, China). All enrolled patients/subjects signed informed consent forms.

Measurement of indicators. Venous blood samples were collected from the fasting NSCLC patients and the patients with benign pulmonary lesions prior to surgery, and the remaining blood samples were collected from routine blood examinations in the healthy subjects. Serum was separated by centrifugation as soon as possible and reserved in a freezer at $-20^{\circ} \mathrm{C}$ until use. Serum OPG and sRANKL levels were detected by enzyme-linked immunosorbent assay (ELISA) according to the manufacturer's instructions. The ELISA kit was purchased from BioVendor (Brno, Czech Republic; the detection sensitivity for OPG and SRANKL were 0.1 and $0.4 \mathrm{pmol} / 1$, respectively).

Statistical analysis. All data were analyzed using SPSS statistical software, version 17.0 (SPS, Inc., Chicago, IL, USA). The category variables are represented by the number of cases, and the significance between two groups was tested using the $\chi^{2}$ test. Quantitative data are presented as the mean \pm standard deviation. The difference between two groups was analyzed by the Mann-Whitney U-test. Meanwhile, the variance in serum sRANKL levels, OPG levels and sRANKL/OPG ratios among the three groups were determined with a one-way analysis of variance. Receiver operating characteristic (ROC) curves were used to evaluate the performance of sRANKL and sRANKL/OPG, and the optimal cut-off points were determined based on Youden's index (12). $\mathrm{P}<0.05$ was considered to indicate a statistically significant difference.

\section{Results}

Clinical parameters. As summarized in Table I, the relevant clinical parameters of the patients with NSCLC were compared against the patients with benign space-occupying pulmonary lesions or the healthy controls. The results showed that there was no significant difference between the NSCLC and control
A

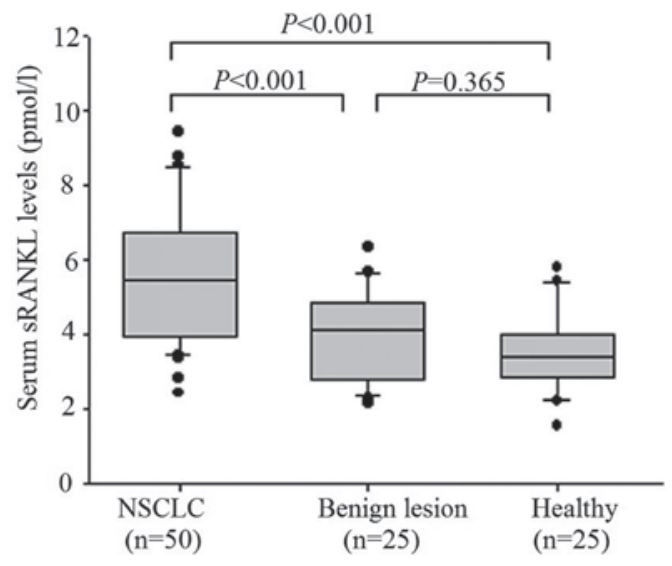

B

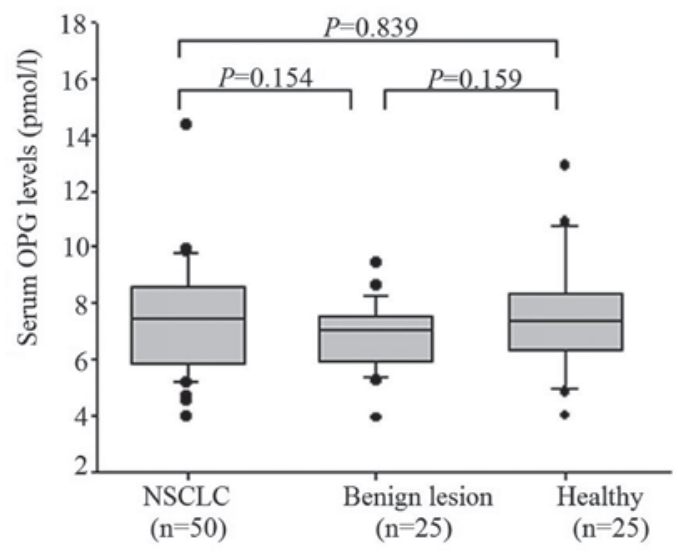

C

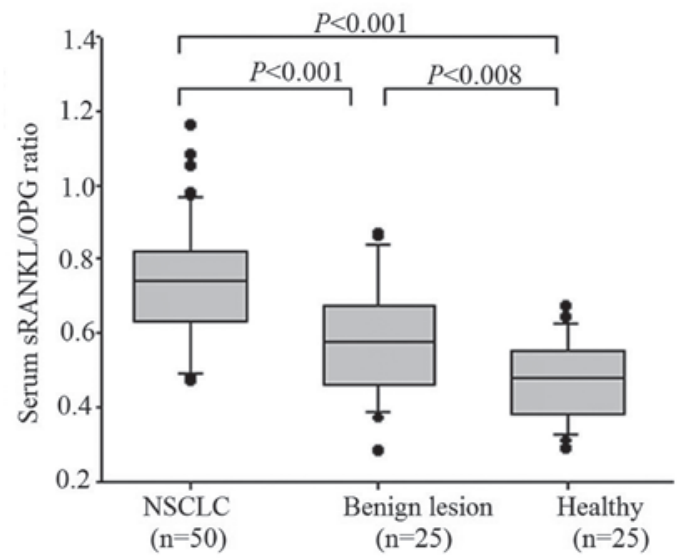

Figure 1. Box plots showing the (A) serum sRANKL level, (B) OPG level and $(C)$ sRANKL/OPG ratio in the patients with NSCLC $(n=50)$, the patients with benign lesions $(n=25)$ and the healthy subjects $(n=25)$. sRANKL, soluble receptor activator of nuclear factor- $\kappa \mathrm{B}$ ligand; $\mathrm{OPG}$, osteoprotegerin.

groups with regard to gender, age or smoking history. The remaining incomparable clinical pathological characteristics of NSCLC are also described in Table I.

Comparison of serum sRANKL and OPG levels, and sRANKL/OPG ratio. As shown in Fig. 1, the serum sRANKL level in the patients with NSCLC $(5.51 \pm 1.75 \mathrm{pmol} / \mathrm{l})$ was significantly higher than that of the patients with benign lesions $(3.98 \pm 1.20 \mathrm{pmol} / \mathrm{l})(\mathrm{P}<0.001)$. Moreover, the sRANKL level of the NSCLC patients was significantly elevated in comparison with the healthy subjects $(3.50 \pm 1.04 \mathrm{pmol} / \mathrm{l})(\mathrm{P}<0.001)$. However, there was no significant difference between the patients with benign lesions and the healthy subjects (Fig. 1A). When analyzing 
Table I. Clinical parameters of the NSCLC group and the combined control group.

\begin{tabular}{|c|c|c|c|}
\hline Category & $\operatorname{NSCLC}(n=50)$ & Controls $(n=50)$ & P-value \\
\hline Gender (male/female), $\mathrm{n}$ & $34 / 16$ & $34 / 16$ & 1.000 \\
\hline Age, years ${ }^{a}$ & $62.4 \pm 10.0$ & $62.8 \pm 15.3$ & 0.878 \\
\hline Positive smoking history, $\mathrm{n}$ & 17 & 13 & 0.383 \\
\hline Pathology & $\begin{array}{l}\text { Adenocarcinoma }(n=30) \\
\text { Squamous cell carcinoma }(n=20)\end{array}$ & $\begin{array}{c}\text { Normal physical } \\
\text { examination }(n=25) \\
\text { Benign space-occupying } \\
\text { lesion }(n=25)\end{array}$ & - \\
\hline Tumor size, $\mathrm{mm}^{\mathrm{a}}$ & $32.1 \pm 17.9$ & - & - \\
\hline Clinical phase (I/II/III), n & $22 / 18 / 10$ & - & - \\
\hline Pathological grading (medium-severe/low), n & $34 / 16$ & - & - \\
\hline Lymph node metastasis (positive/negative), $\mathrm{n}$ & $25 / 25$ & - & - \\
\hline
\end{tabular}

${ }^{a}$ Results are represented as the mean standard \pm deviation. NSCLC, non-small cell lung cancer.

Table II. Efficacy of sRANKL level and sRANKL/OPG ratio in the diagnosis of non-small cell lung cancer using an ROC.

\begin{tabular}{|c|c|c|c|c|c|}
\hline Indicator & Cut-off & $\operatorname{AUC}(95 \% \mathrm{CI})$ & Sensitivity, $\%$ & Specificity, \% & Accuracy, $\%$ \\
\hline sRANKL & $4.20 \mathrm{pmol} / \mathrm{l}$ & $0.837(0.746-0.927)$ & 74.0 & 84.0 & 77.3 \\
\hline sRANKL/OPG & 0.60 & $0.922(0.863-0.980)$ & 84.0 & 88.0 & 85.3 \\
\hline
\end{tabular}

sRANKL, soluble receptor activator of nuclear factor- $\mathrm{kB}$ ligand; OPG, osteoprotegerin; ROC, receiver operating characteristic curve; AUC, area under the ROC curve; CI, confidence interval.

the differences in serum OPG levels, there was no statistical significance among the three groups; the values for the patients with NSCLC, the patients with benign lesions and the healthy subjects were $7.43 \pm 1.86,6.81 \pm 1.20$ and $7.52 \pm 2.03 \mathrm{pmol} / \mathrm{l}$, respectively (Fig. 1B). Whereas the sRANKL/OPG ratio was increased in the patients with NSCLC $(0.74 \pm 0.16)$ in comparison to the patients with benign lesions $(0.59 \pm 0.16, \mathrm{P}<0.001)$ and the healthy subjects $(0.47 \pm 0.11, \mathrm{P}<0.001)$. When comparing the sRANKL/OPG ratio in the patients with benign lesions and the healthy subjects, no significant differences were found (Fig. 1C).

Efficacies of serum SRANKL level and $s R A N K L / O P G$ ratio in the diagnosis of NSCLC. To further determine the diagnostic significance of the sRANKL level and the sRANKL/OPN ratio, ROC curves were used to analyze the efficacy in discerning NSCLC patients from those with benign lesions or the healthy controls. As shown in Table II, based on the greatest Youden's index, the optimal cut-off points for sRANKL level and sRANKL/OPG ratio were $4.20 \mathrm{pmol} / 1$ and 0.60 , respectively. The area under the curve (AUC) for sRANKL level and SRANKL/OPG ratio was 0.837 [95\% confidence interval (CI), 0.746-0.927] and 0.922 (95\% CI, 0.863-0.980), respectively. The sensitivity, specificity and accuracy for sRANKL levels at the optimal cut-off point were 74.0, 84.0 and 77.3\% respectively. Furthermore, the performance of the sRANKL/OPG ratio was better than the SRANKL levels in the diagnosis of NSCLC. The sensitivity, specificity and accuracy for the sRANKL/OPG ratio was $84.0,88.0$ and $85.3 \%$, respectively.
Diagnostic significance of serum sRANKL level and sRANKL/OPG ratio in distinguishing between benign and malignant space-occupying pulmonary lesions. In addition, it was difficult to distinguish between the patients with malignant lesions and those with benign lesions. The present study also analyzed the diagnostic efficacy of the sRANKL level and the sRANKL/OPG ratio in discerning malignant and benign lesions. Based on the greatest Youden's index, the optimal diagnostic cut-off point for the sRANKL level and the sRANKL/OPG ratio were $5.24 \mathrm{pmol} / 1$ and 0.63 , respectively (Table III). The AUC for each indictor was 0.744 (95\% CI, 0.632-0.855) and 0.751 (95\% CI, 0.631-0.871), respectively. The sensitivity, specificity and accuracy of the sRANKL level in the diagnosis of benign or malignant pulmonary lesions were $66.0,84.0$ and $68.0 \%$, respectively. With regard to the $\mathrm{sRANKL/OPG}$ ratio, the sensitivity, specificity and accuracy were 78.0, 64.0 and $73.3 \%$, respectively. Therefore, the sRANKL/OPG ratio exhibited a better performance compared with the sRANKL level.

\section{Discussion}

Conventional diagnostic markers, such as carcinoembryonic antigen (CEA) and CA199, have limited sensitivity (CEA, 52\%; CA199, 48.2\%) and specificity (CEA, 80\%; CA199, 90\%) with regard to discerning patients with lung cancer, particularly those with advanced stage (13). Although patients who undergo a surgical resection are able to achieve a favorable clinical 
Table III. Efficacy of serum sRANKL level and sRANKL/OPG ratio in distinguishing between benign and malignant space-occupying pulmonary lesions using an ROC.

\begin{tabular}{lccccc}
\hline Indicator & Optimal cut-off point & AUC $(95 \% \mathrm{CI})$ & Sensitivity, $\%$ & Specificity, \% & Accuracy, \% \\
\hline sRANKL & $5.24 \mathrm{pmol} / 1$ & $0.744(0.632-0.855)$ & 60.0 & 84.0 & 68.0 \\
sRANKL/OPG & 0.63 & $0.751(0.631-0.871)$ & 78.0 & 64.0 & 73.3 \\
\hline
\end{tabular}

sRANKL, soluble receptor activator of nuclear factor- $\mathrm{KB}$ ligand; OPG, osteoprotegerin; ROC, receiver operating characteristic curve; AUC, area under the ROC curve; CI, confidence interval.

outcome, it is impossible to avoid cancer metastasis and the associated poor outcome, which greatly reduces the middle and long-term patient survival rates. Hence, there has been an urgent requirement to identify effective diagnostic approaches. Recently, following the development of molecular biology, studies have begun to investigate convenient or inexpensive markers to aid in the diagnosis and prognosis of malignant tumors (14). Tumor markers are those molecules that cancer cells secrete into the body fluids or tissue. Generally, the levels of these biomarkers are significant different in comparison with healthy controls. Clinicians can use these markers to diagnose patients with cancer, monitor tumor progress, guide treatment, monitor recurrence or metastasis, and predict prognosis. Currently, CEA, neuron-specific enolase, carbohydrate antigen 125 (CA-125) and CA19-9 have been applied in the clinic to diagnose or monitor the progress of lung cancer, with limited success (15). A long-term observation of these markers revealed that none of them were able to accurately determine the patient's disease state (16).

As aforementioned, the roles of OPG and RANKL have been extensively investigated in the regulation of tumor progression $(16,17)$. The two molecules were shown to be significant in bone regulation (18). More importantly, OPG and RANKL were indicated to be involved in cancer pathogenesis, development and metastasis $(9,10)$. Furthermore, the RANKL/OPG ratio was shown to exhibit a positive correlation with NF- $\mathrm{\kappa B}$ activation (19). This finding suggested that OPG/RANKL activated NF- $\mathrm{kB}$, which may in turn stimulate a series of signaling pathways. Thereby, RANKL and OPG could regulate cell differentiation, function or apoptosis. In view of this, either RANKL or OPG would promote the occurrence of NSCLC. However, these hypotheses require confirmation in future studies.

In the present study, the serum sRANKL and OPG levels were initially determined. The sRANKL level and sRANKL/OPG ratio were indicated to be potential NSCLC biomarkers. With the exception of the aforementioned studies, few studies have been published that are associated with RANKL or OPG and the progression of lung cancer. The present study found that the sRANKL levels were significantly higher in the NSCLC patients compared with the healthy controls or patients with lung benign space-occupying lesions. In accordance with these results, the study by Karapanagiotou et al also demonstrated higher sRANKL levels in NSCLC patients (20). However, there was no difference in the OPG levels among the three groups in the present study. This finding was not consist with the results in the study by Karapanagiotou et al, where it was concluded that higher OPG levels promoted the development of lung cancer metastasis (20). This may be due to the relatively small sample size resulting in selective bias. Moreover, the present study and that of Karapanagiotou et al demonstrated that the sRANKL/OPG ratio was significantly higher in the NSCLC patients. All these findings indicated that sRANKL or OPG promoted the progression of cancer. Another previous study proved that blocking OPG or attenuating sRANKL/OPG inhibited tumor cell proliferation (21).

Significantly, the present study identified that the sRANKL level and the sRANKL/OPG ratio were potential biomarker candidates for lung cancer. According to the ROC curve analysis, the sensitivity, specificity and accuracy of the indictors exhibited superior performance in comparison with previously reported clinical lung cancer biomarkers, including CEA and CA19-9 (none of which were specific biomarkers of lung cancer) (22). The AUC values for the sRANKL level and the sRANKL/OPG ratio were 0.837 and 0.922 , respectively. Additionally, the sRANKL level and the sRANKL/OPG ratio exhibit potential as biomarkers with the ability to discern between malignant or benign lesions. Clinically, the majority of lung cancer patients show no signs of disease in the early stages. For instance, certain patients with lung nodules have atypical imaging pictures or a tumor diameter $<2.5 \mathrm{~cm}$ (23). The present study also analyzed the performance of the sRANKL level and the sRANKL/OPG ratio in the diagnosis of malignant versus benign lesions. To date, few studies have proposed any available factors to apply in the clinic. However, the present study only determined the performance of the sRANKL level and the sRANKL/OPG ratio in relatively small groups, therefore, further analysis will be required as a large-scale validation that will also include the comparison of the sRANKL level and the sRANKL/OPG ratio against other currently used markers, such as CEA or CA19-9.

In summary, the serum sRANKL level and the sRANKL/OPG ratio may have potential as novel biomarkers for diagnosis of NSCLC. OPG and RANKL may be involved in the pathogenesis of NSCLC. The present results may provide important biomarker candidates for early diagnosis of NSCLC.

\section{References}

1. Jemal A, Bray F, Center MM, Ferlay J, Ward E and Forman D: Global cancer statistics. CA Cancer J Clin 61: 69-90, 2011.

2. Wang Y, Gu J, Roth JA, Hildebrandt MA, Lippman SM, Ye Y, Minna JD and Wu X: Pathway-based serum microRNA profiling and survival in patients with advanced stage non-small cell lung cancer. Cancer Res 73: 4801-4809, 2013.

3. Ourari-Dhahri B, Ben Slima H, Ben Amar J, El Gharbi L, Ali M, Baccar Azzabi S, Aouina $\mathrm{H}$ and Bouacha $\mathrm{H}$ : Management of non small cell lung cancer. Tunis Med 90: 847-851, 2012 (In French). 
4. Kuner R, Muley T, Meister M, Ruschhaupt M, Buness A, Xu EC, Schnabel P, Warth A, Poustka A, Sültmann H and Hoffmann $\mathrm{H}$ : Global gene expression analysis reveals specific patterns of cell junctions in non-small cell lung cancer subtypes. Lung Cancer 63: 32-38, 2009.

5. Esteban E, Casillas M and Cassinello A: Pemetrexed in first-line treatment of non-small cell lung cancer. Cancer Treat Rev 35: 364-373, 2009.

6. Peng X, Guo W, Ren T, Lou Z, Lu X, Zhang S, Lu Q and Sun Y: Differential expression of the RANKL/RANK/OPG system is associated with bone metastasis in human non-small cell lung cancer. PLoS One 8: e58361, 2013.

7. Kong YY, Feige U, Sarosi I, Bolon B, Tafuri A, Morony S, Capparelli C, Li J, Elliott R, McCabe S, et al: Activated T cells regulate bone loss and joint destruction in adjuvant arthritis through osteoprotegerin ligand. Nature 402: 304-309, 1999.

8. Harada $\mathrm{S}$ and Takahashi N: Control of bone resorption by RANKL-RANK system. Clin Calcium 21: 1121-1130, 2011 (In Japanese).

9. Hanada R, Hanada T, Sigl V, Schramek D and Penninger JM RANKL/RANK-beyond bones. J Mol Med (Berl) 89: 647-656, 2011.

10. Peters S and Meylan E: Targeting receptor activator of nuclear factor-kappa B as a new therapy for bone metastasis in non-small cell lung cancer. Curr Opin Oncol 25: 137-144, 2013.

11. Travis WD, Brambilla E, Noguchi M, Nicholson AG, Geisinger KR, Yatabe Y, Beer DG, Powell CA, Riely GJ, Van Schil PE, et al: International association for the study of lung cancer/american thoracic society/european respiratory society international multidisciplinary classification of lung adenocarcinoma. J Thorac Oncol 6: 244-285, 2011.

12. Fluss R, Faraggi D and Reiser B: Estimation of the Youden Index and its associated cutoff point. Biom J 47: 458-472, 2005.

13. Diamandis EP, Goodglick L, Planque $C$ and Thornquist MD: Pentraxin-3 is a novel biomarker of lung carcinoma. Clin Cancer Res 17: 2395-2399, 2011.
14. Wiedemann GJ: Biomarker screening for early detection of cance. Dtsch Med Wochenschr 138: 43-45, 2013 (In German).

15. Ferrigno D, Buccheri G and Giordano C: Neuron-specific enolase is an effective tumour marker in non-small cell lung cancer (NSCLC). Lung Cancer 41: 311-320, 2003.

16. Strathmann FG, Schulte S, Goerl K and Petron DJ: Blood-based biomarkers for traumatic brain injury: Evaluation of research approaches, available methods and potential utility from the clinician and clinical laboratory perspectives. Clin Biochem 47: 876-888, 2014.

17. Ibrahim T, Sacanna E, Gaudio M, Mercatali L, Scarpi E, Zoli W, Serra P, Ricci R, Serra L, Kang Y and Amadori D: Role of RANK, RANKL, OPG and CXCR4 tissue markers in predicting bone metastases in breast cancer patients. Clin Breast Cancer 11: 369-375, 2011.

18. Dougall WC and Chaisson M: The RANK/RANKL/OPG triad in cancer-induced bone diseases. Cancer Metastasis Rev 25: 541-549, 2006

19. Owen S, Ye L, Sanders AJ, Mason MD and Jiang WG: Expression profile of receptor activator of nuclear- $\kappa \mathrm{B}$ (RANK), RANK ligand (RANKL) and osteoprotegerin (OPG) in breast cancer. Anticancer Res 33: 199-206, 2013.

20. Karapanagiotou EM, Terpos E, Dilana KD, Alamara C, Gkiozos I, Polyzos A and Syrigos KN: Serum bone turnover markers may be involved in the metastatic potential of lung cancer patients. Med Oncol 27: 332-338, 2010.

21. Silva I and Branco JC: Rank/Rankl/opg: Literature review. Acta Reumatol Port 36: 209-218, 2011.

22. Shu J, Li CG, Liu YC, Yan XC, Xu X, Huang XE, Cao J, Li Y, $\mathrm{Lu}$ YY, Wu XY, et al: Comparison of serum tumor associated material (TAM) with conventional biomarkers in cancer patients. Asian Pac J Cancer Prev 13: 2399-2403, 2012.

23. Prisadov GT, Uchikov AP, Welker K, Wallimann $H$, Murdzhev KA and Uzunova VN: Size of tumour as a risk factor for malignancy in patients with peripheral pulmonary nodules. Folia Med (Plovdiv) 54: 17-21, 2012. 